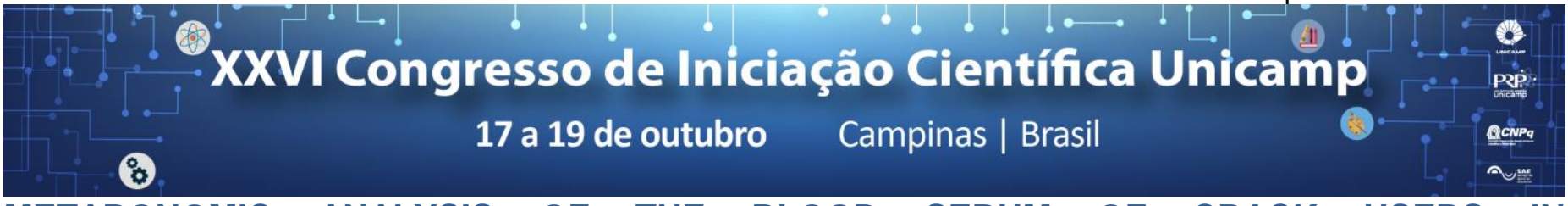

\title{
METABONOMIC ANALYSIS OF THE BLOOD SERUM OF CRACK USERS IN RESOCIALIZATION PROCESS
}

\section{Pedro H. B. Pinto*, Tássia B. B. C. Costa, Ljubica Tasic}

\begin{abstract}
Crack-cocaine is a potent form of cocaine abuse and is considered, globally, as one of the most potentially dangerous illicit drugs. In Brazil, it is one of the most commonly used drugs because it is cheap and its effects are very quick. The identification of biomarkers in crack-cocaine users may add-in the search for new treatments for this drug addicts. This project aims a metabonomics study by ${ }^{1} \mathrm{H}$ NMR on blood serum samples from healthy and crack-cocaine dependent women. ${ }^{1} \mathrm{H}$ NMR spectral data were analyzed by statistical analysis techniques, such as principal component analysis (PCA), partial least squares discriminant analysis (PLS-DA) and sparse PLS-DA (sPLS-DA). Subsequently, important biomarkers for crack-cocaine abuse were identified.
\end{abstract}

\section{Key words:}

${ }^{1} H$ NMR, metabonomics, crack-cocaine

\section{Introduction}

Crack, a psychoactive drug, is a huge concern for the public health in Brazil, the world greatest consumer of this illicit drug. In the long term, the constant use of crack brings changes in attention tests, verbal fluency, visual memory and executive functions associated directly to neuropsychological deficits. The project targets to find metabolites that differentiate healthy volunteers from crack users, through the application of ${ }^{1} \mathrm{H} N M R$ spectroscopy of serum samples, to distinguish the samples in three groups: control $(\mathrm{HC})$, user of crack before the treatment (1) and users of crack after treatment (2) and also to identify biomarkers associated with crack dependence and treatment.

\section{Results and Discussion}

The ${ }^{1} \mathrm{H}-\mathrm{NMR}$ spectra were normalized by the sum and mean centered. The mean spectra of each group were obtained and the subtraction between them was executed with success.

From Image 1 it is possible to realize that there are differences in metabolites concentrations in the mean spectra of the groups.

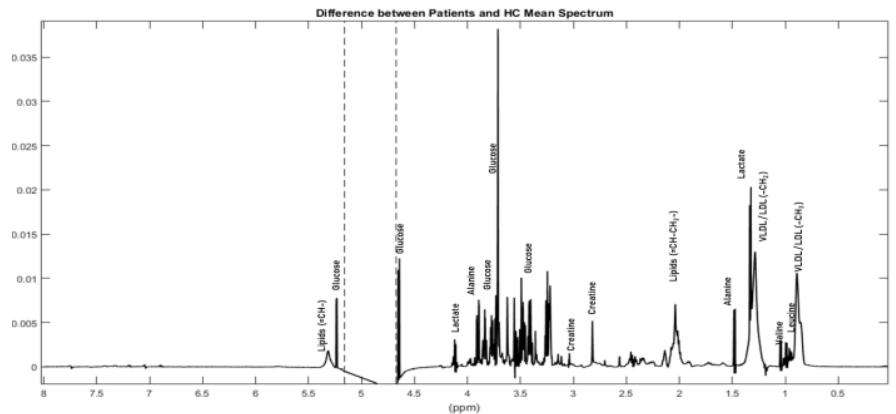

Image 1. Spectral differences among the mean control group ${ }^{1} \mathrm{H}-\mathrm{NMR}$ subtracted from the mean crack users' ${ }^{1} \mathrm{H}$ NMR spectra before treatment.

It has been observed that crack users have higher concentrations of lipids, sugars, creatine, lactate and some amino acids, such as L-alanine, L-valine and Lleucine.

From the multivariate analysis, we got conclusive results, as from sPLS-DA. But, none of the latent variables separated group 1 and grouped groups 2 and $\mathrm{HC}$, suggesting that there are no great similarities between treated patients and healthy volunteers.

From Image 2, it is possible to see that the first component separated the treated patients (green) from the other two groups (patients before treatment - red and healthy volunteers - blue), and the most important loadings (NMR chemical shifts) were then compared to database.

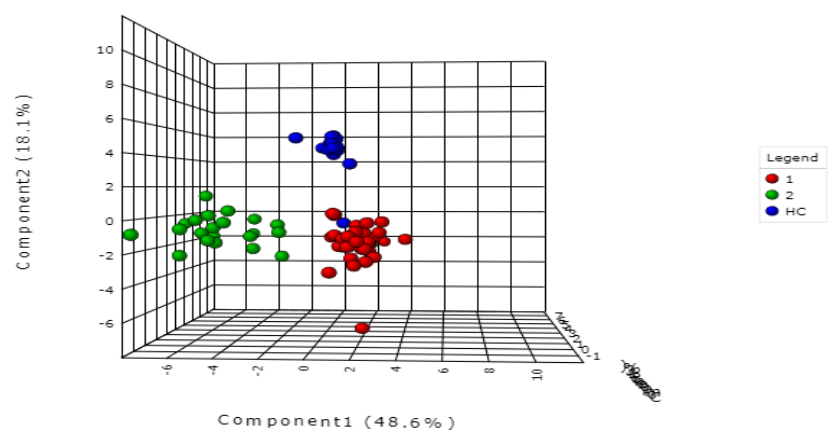

Image 2. Image of scores of the sPLS-DA models

By the loadings, we identified possible biomarkers such as: L-glutamate, L-proline, L-glutamine, L-aspargine, Lvaline, D-glucose, and glycerol.

\section{Conclusions}

As the samples are human blood serum ones - they make the pattern recognition analyzes very complex since each person has a different life history with a different amount of drug consumption and all of these can affect the metabolites present in the serum. However, the results presented so far are satisfactory in what is related to the search for biomarkers that differentiate the control group from the crack users' groups and biomarkers associated with crack dependence and treatment.

FAPESP, CNPq and SAE

\section{Acknowledgement}

${ }^{1}$ Tasic, L. et al. NMR Spectroscopy Metabolomics Applied to Crack Cocaine Users and Patients with Schizophrenia: Similar Behavior but Different Molecular Causes. ChemistrySelect 2, 2927-2930 (2017).

2 Dinis-Oliveira, R. J. Metabolomics of cocaine: implications in toxicity. Toxicol. Mech. Methods 25, 494-500 (2015).

3 Costa, T. Metabonômica Aplicada ao Diagnóstico e Estadiamento de Doenças Hepáticas. Dissertação (2016). 